\title{
Nachlese zum Kongress: Wegweisende Aspekte der 62. Jahrestagung der Deutschen Gesellschaft für Phlebologie in Leipzig
}

Die 62. Jahrestagung der Deutschen Gesellschaft für Phlebologie in Leipzig fand während der Corona-Pandemie statt. Sie wurde aufgrund des konsequent umgesetzten $\mathrm{Hy}$ gienekonzepts ein großer Erfolg: 730 Teilnehmer waren vor Ort, darüber hinaus nahmen weitere 180 Teilnehmer per Livestream-Format an der Tagung teil. Die Erfahrungen dieser von Dr. Tobias Hirsch als Tagungspräsident erfolgreich durchgeführten Tagung lassen uns folgende Lehren ziehen:

1. Kompakte kursartige Formate wie der Crashkurs, das Update Phlebologie, Handson-Workshops, das Weiterbildungsforum und Firmensymposien waren gut besucht. Dementsprechend werden wir auch in $\mathrm{Zu}$ kunft derartige praxisrelevante Formate anbieten.

2. Erstmals gab es in Leipzig einen konsequent durchgeführten internationalen Vortragsstrang mit Simultanübersetzung. Das Interesse ausländischer Phlebologen, als Zuhörer oder auch als Referent auf unserer deutschen Jahrestagung dabei zu sein, war unerwartet groß. Offenbar genießt die deutsche Phlebologie international eine große Reputation. Dies wollen wir auch auf zukünftigen Jahrestagungen ausbauen. Die Jahrestagung in Leipzig hat gezeigt, wie spannend es ist, die international nicht selten deutlich abweichenden phlebologischen Vorgehensweisen und Ansichten kennenzulernen und die in Deutschlang praktizierte Phlebologie auf diese Art und Weise auf den Prüfstand zu stellen.

3. Auch die Angebote für nichtärztliche Medizinalberufe waren trotz Corona-Pandemie sehr gut besucht. Der Kurs für PhlebologieAssistenten, der sich vor allem an medizinische Fachangestellte richtet, war ausgebucht. Die Teilnehmerzahl des Updates Wundmanagement konnte mehr als verdoppelt werden. Auch auf den zukünftigen Jahrestagungen wollen wir nicht nur Ärztinnen und Ärzte, sondern alle Professionen, die sich mit Phlebologie beschäftigen, ansprechen. Gerade für die Veranstaltung „Update Wundmanagement“ gehen wir von einer weiteren starken Steigerung der Teilnehmerzahl aus.
4. Der fachliche Austausch im persönlichen Gespräch war auch in Leipzig wieder sehr intensiv und gewinnbringend für alle Teilnehmer vor Ort. Andererseits hat die digitale Kongressvariante unter bestimmten Gesichtspunkten besondere Vorteile, sodass wir auch zukünftig Hybridkongresse anstreben wollen.

5. In Krisenzeiten zeigt sich besonders, inwieweit man sich auf Partner verlassen kann. Wir sind ausgesprochen dankbar für die große Unterstützung der Partner aus der Industrie, unserer Kongressagentur wikonect und der betreuenden Technikfirma Röll Media und freuen uns auf eine weitere vertrauensvolle Zusammenarbeit.

Entsprechend dem Kongressmotto „Gemeinsam in Leipzig: Phlebologie national und international“ ist die phlebologische Gemeinschaft in Leipzig weiter gewachsen und gereift. In dieser Gemeinschaft sind wir auch in Zukunft bestens aufgehoben.

Prof. Dr. Markus Stücker

Präsident der Deutschen Gesellschaft für Phlebologie
IMPRESSUM

Verantwortlich für Mitteilungen der DGP:

Dr. med. Erika Mendoza 